\title{
Über Sedimentuntersuchungen eiweißloser Harne bei therapeutischer Quecksilberapplikation (Inunktionskuren).
}

Von

Dr. Richard Fischel (Bad Hall).

Die erst jüngst von Lüthje festgestellte Tatsache, daß das salizylsauere Natron an sich und in seinen Zusammensetzungen Cylindrurie ohne Eiweißausscheidung hervorzurufen vermöge, veranlaßte mich zu untersuchen, ob das als Nierengift experimentell und klinisch genau studierte $\mathrm{Hg}$ auch in therapeutischen Dosen Veränderungen hervorrufen könne, die im Harnsedimente ihren Ausdruck finden.

Ein Rückblick auf die spärlichen Literaturangaben ergibt, daß $F$ ürbringer als erster systematisch den Harn während der verschiedenen Formen der Hg-Darreichung geprüft hat und in $8 \%$ seiner Fälle eine höchstens $1 / 3 \%$ erreichende Albuminurie konstatieren konnte. Das Sediment wurde noch durch Filtration des fast durchwegs klaren Harns gewonnen; von Formbestandteilen waren nur "berzlich wenig" nachzuweisen: hyaline Cylinder in schwankender Zahl, spärliche Rundzellen (Schleimkörperchen? Lymphkörperchen?) vielleicht Nierenepithelien, gr. Plattenzellen der Blase, niemals rote Blutkörperchen, niemals epitheliale oder dunkelkörnige Cylinder.

Aber erst Welander war es durch Benützung der S tenbeks chen Zentrifuge vorbehalten, die von $\mathrm{F} u ̈ \mathrm{rbr}$ inger 
angebahnten Untersuchungen zu erweitern und die ganze Frage auf eine neue Basis zu stellen. Er fand in zwei Untersuchungsreihen, ${ }^{1}$ ) daß das angewandte $\mathrm{Hg}$ in $70 \%$ Cylindrurie erzeugte, der nur zum geringsten Teile eine meist höchst unbedeutende Albuminurie entsprach.

Nach Schwimmer hängt die in $9 \cdot 2 \%$ während der $\mathrm{Hg}$ Behandlung auftretende Albuminurie vom luetischen Prozesse ab. Nur in einem Falle legt er die nach der dritten Sozojodolinjektion bei einem kräftigen 25jährigen Manne in Erscheinung tretende Ausscheidung von Eiweil, granul. und hyal. Cylindern dem Medikament zur Last.

Die von Welander erhaltenen Resultate führt er auf die starke Häufung der von jenem geübten Thymolquecksilberinjektionen (3-4 wöchentlich) zurück, eine Auffassung, die, wie W el and er selbst hervorhebt, auf einem Irrtum S ch w i m mer s beruht.

$\mathrm{H}$ eller hat auf der Lewinschen Klinik Vergleiche über den Einfluß des $\mathrm{Hg}$ auf die Nieren zwischen Inunktionsund $1 \%$ Sublimatinjektionskuren angestellt:

Unter 28 inungierten Fällen 9mal Albumen $-24 \%$.

$$
\text { "241 injizierten } " 9, \quad \text { " } 3.7 \% \text {. }
$$

Bei der ersten Behandlungsform war die Albuminurie zweimal mit Cylindrurie verbunden. Eine vorher stets gesunde Frau akquirierte nach 30 Einreibungen eine mittelschwere hämorrhagische Nephritis. Bei der zweiten Versuchsreihe war nur in einem Fall bei einer Patientin, die vorher schon eine Nephritis durchgemacht hatte, nach 16 Injektionen Albumen $1 / 3 \%$ und eine Ausscheidung von Cylindern aufgetreten.

Karwonen teilt mit, daß die Erfahrungen Holméns vollkommen im Einklang mit denen Welanders stehen. Unter 32 Fällen war im albumenfreien Harn $4 \mathrm{mal}$ ein positiver Cylinderbefund erhoben worden. Als Reaktion auf Eiweiß kam nur die Kochprobe zur Anwendung. In seiner Privatpraxis hat Karwonen viermal Albuminurie und Cylindrurie beobachtet. Immer aber zeigte sich ein positives Resultat erst nach mehr

1) In der zweiten Reihe erstrecken sich die Untersuchungen auf 180 fortlaufend und 42 nur zeitweilig beobachtete Patienten. 
als 20 Einreibungen, wie aus den beigefügten Krankengeschichten ersichtlich ist.

In Unnas Monatsheften fand ich noch ein Referat, ${ }^{1}$ ) dem zufolge ein "ungenannter Verfasser" über Welanders Untersuchungen berichtet, in dem aber eigener Untersuchungen des Anonymus keine Erwägung getan wird.

Es sei auch auf die Experimente Gravagnas ${ }^{1}$ ) Rücksicht genommen, die, nach Applikation therap. Dosen, path. anat. ein vollständig negatives Resultat für die Kaninchenniere ergaben. Auch bei 60 Patienten konnte in keinem einzigen Falle Albuminurie oder Ausscheidung geformter Elemente nachgewiesen werden.

Karwonea zitiert eine Arbeit Overbecks $\mathrm{s}^{2}$ ) aus dem Jahre 1860, der von einem "desquamierenden Katarrh der Nierenkanälchen" spricht, mit der Annahme, es handle sich um eine gewöhnliche aszendierende katarrhalische Reizung der Nieren. kelche und Harnkanälchen.

Resümieren wir, so ist Welander der einzige Autor, der in exakter Weise den Nachweis einer hyalinen Cylindrurie in eiweißlosen Harnen erbrachte und es muß mit Verwunderung erfüllen, daß bei der großen Verbreitung der Hg-Kuren eine weitere Beschäftigung mit der gewiß nicht bedeutungslosen Frage ausblieb.

\section{Untersuchungstechnik.}

L üthje konnte erst durch eine äußerlich unscheinbare, aber durch den Erfolg als wesentlich sich darstellende Modifikation der Sedimentgewinnung den Einfluß der Salizylpräparate auf die Niere klarlegen. Das Verfahren beschreibt er folgendermaßen: "Mit einem Ballonsanger wurde aus der Tiefe des Uringlases, da wo sich die Nubecula gebildet hatte, eine gewisse Menge Urin entnommen und dann zentrifugiert. In anderen Fällen wurden die oberen Schichten des Harnes abgegossen und dann die Nubecula enthaltenden Partien zentrifugiert. Ich

1) Die Originalmitteilung war mir nicht zugänglich.

2) Die Arbeit war mir im Original nicht zugänglich. 
betone das ausdrücklich. Wer diesen Modus nicht einhält und den Urin aus den oberen Schichten des Uringlases nimmt, dem werden Cylinder und sonstige Formbestandteile oft entgehen."

Ich habe zumeist den letzteren Modus als den einfacher ausführbaren vorgezogen, und nur wenn die Nubecula sich schwebend erhielt, zu ihrer Übertragung ins Zentrifugiergläschen die Ballonpipette benutzt.

Der über die Nacht sezernierte Harn wurde von den Patienten des Morgens in ca. $600 \mathrm{~cm}^{3}$ fassende Spitzgläser, die peinlichst gereinigt und vor dem Gebrauch sterilisiert wurden, gelassen und die Nubecula nach ihrem Absetzen um ca. $3 \mathrm{Uhr}$ Nachmittags zentrifugiert. Das becherförmige Zentrifugiergläschen konnte ca. $50 \mathrm{~cm}^{3}$ der Nubecula aufnehmen, deren Formelemente dann mittels einer durch einen Elektromotor angetriebenen Zentrifuge ${ }^{1}$ ) mit ca. $6000 \mathrm{Um} \mathrm{drehungen}$ in der Minute (durch den Tourenzähler kontrolliert) ausgeschleudert wurden, wozu durchschnittlich 15-20 Minuten genïgten, um einen deutlichen Bodensatz zu erhalten. Manche Harne mußten bis 24 Stunden und noch länger stehen, bis eine deutliche und dann oft noch spärliche Nubeculabildung eintrat. In seltenen Fällen kam es überhaupt zu keiner Nubeculabildung. Auf die Varietäten derselben und ihrer Beziehung zur Sedimentgewinnung weist schon Gentzen hin.

Das erhaltene Sediment stellte bald eine dicke Gallerte, bald nur in Flüssigkeit suspendierte Flöckchen dar, mit den mannigfaltigsten Übergängen zwischen diesen beiden Extremen. Aus äußeren Gründen war ich manchmal gezwungen, die klinische Zentrifuge mit Wasserantrieb und bedeutend geringerer Tourenzakl zu benutzen, deren Zentrifugiergläschen nur mit ca. $10 \mathrm{~cm}^{3}$ Flüssigkeit gefüllt werden können. Ich brauche wohl nicht erst hervorzuheben, daß der Vergleich der mikroskopischen Präparate bezüglich der Zahl der ausgeschleuderten Formelemente einer Nubecula, die auf jeder der beiden Zentrifugen verarbeitet wurde, zu Gunsten der "elektrischen" Zentrifuge ausfiel.

1) Für die Er]aubnis zur Benützung der Zentrifuge gestatte ich mir Herrn Prof. Gad, dem Vorstand des physiologischen Institutes, seinem Assistenten Herrn Doz. Kahn für das freundliche Entgegenkommen meinen besten Dank zu sagen. 
Die zur Übertragung auf den Objektträger verwendeten Pipetten wurden nur zur einmaligen Untersuchung eines Sedimentes benützt und dann exakt gereinigt und sterilisiert.

Der Urin der Weiber wurde durch Kathetrisieren gewonnen. Der Morgenharn wurde herangezogen, um bei allen Individuen gleiche Versuchsbedingungen zu setzen, dann aber auch um eventuell intermittierende Albuminurien auszuschalten

Der Vor- oder Nachmittagsharn wurde in einigen Fällen untersucht und schien es (system. diesbezügliche Untersuchungen habe ich leider unterlassen), als ob der Morgenharn an Formelementen reichlicher wäre als die Tagesportionen. (Reichlichere Nubeculabildung des Morgenharns?)

Die Untersuchungen sind ausschließlich an klinischen Patienten ausgeführt, da bei ambulanten Kranken die Komplikation unkontrollierbarer Exzesse (in bacho etc.) (Glaser) eine Verschleierung der Resultate hätte zur Folge haben können.

Von einem Sedimente wurden mindestens zwei mikroskopische Präparate genau durchmustert, bei negativem Ausfall vier und mehr.

\section{Eigene Versuchsergebnisse.}

Vor allem mußte festgestellt werden, ob die Syphilis an sich in den einzelnen zur Bebandlung kommenden Stadien Cylindrurie ohne Eiweißausscheidung hervorzubringen im stande ist. In Berücksichtigung der Resultate von Klieneberger, Oxenius und ron Gentzen, denen zufolge gesunde, nicht fiebernde Individuen in $89 \cdot 24 \%$ Cylinder überhaupt, granulierte Cylinder in $4.3 \%$ bzw. in $59.5 \%$ Cylinder überhaupt, granulierte Cylinder in $36.14 \%$ im Harnsedimente aufwiesen, wobei nicht vergessen werden darf, daß die ersterwähnten Autoren den Morgenharn, der zweite Autor den in der Sprechstunde gelassenen Harn untersuchte, mußte, falls es eine luetische Cylindrurie gibt, erwartet werden, daßj bei Syphilitikern die Zahl der bei der eben besprochenen "physio]ogischen Cylindrurie" ausgeschiedenen Elemente eine pathologische Steigerung erfahre.

Zur Orientierung wurde von drei gesunden, im Alkoholgenuß mäßigen Kollegen der Ḱlinik der Harn untersucht (bei zweien 
der Morgen-, bei einem der Vormittagsharn). Bei allen fand sich je ein hyaliner Cylinder (bei einem Kollegen bei zweimaliger Untersuchung) beidemal.

Für die oben angezogene Frage kommen nur 13 Fälle (Männer) des gesamten Untersuchungsmateriales in Betracht. Davon hatten 6 keine Cylinder, 7 einen bis zwei hyal. Cylinder. Der Grund, warum nur 13 Patienten in die Beobachtungsreihe aufgenommen wurden, liegt darin, daß die Patienten gleich oder sehr bald nach dem Spitalseintritt in Behandlung genommen werden mußten. Zeigten sich nun am zweiten oder dritten Tage nach anfänglich negativem Resultate wenn auch nur spärliche Cylinder, so konnten diese bereits einer durch $\mathrm{Hg}$ bewirkten Nierenreizung ihre Entstehung verdanken. Der von Klieneberger und Oxenius geübte Modus zur Konstatierung einer phys. Cylindrurie, durch 4 Tage das Sediment zu untersuchen, konnte nicht ausgeführt werden.

Wie schwierig es aber ist, negative Resultate zu verwerten, zeigt folgender Versuch (Fall Nr. 5): In dem mittels elektrischer Zentrituge gewonnenen Sedimente war in vier Präparaten kein Cylinder nachweisbar. Das nach Zusatz einiger Chloroformtropfen über Nacht aufbewahrte Sediment wird am nächsten Morgen mit der klinischen Wasserzentrifuge nochmals zentrifugiert und so noch mehr konzentriert. Jetzt fanden sich sofort im ersten angefertigten Präparat 2 hyal. Cylinder.

In einem der nächsten Tage trat der umgekehrte Fall ein. In dem durch elektr. Zentrifuge gewonnenen Sediment war sofort im ersten Präparat ein hyaliner Cylinder zu konstatieren. In dem nochmals mit der klinischen Zentrifuge konzentrierten Sediment in 4 Präparaten: kein cylindrisches Gebilde.

Sämtliche Patienten bis auf einen (positiven), der sich in der gummösen Phase befand, waren im Stadium der ersten Eruption oder im Ausbruchsstadium eines Rezidivs.

Welanders Zahlen, die dadurch, daß sie für frisch gelassenen Harn gelten also nach weniger vollkommener Methode an sofort nach der Entleerung centrif. Harn gewonnen sind, prozentuell hinter meinen zurückstehen, ergeben: 


\section{Reihe.}

1. Ausbruch der Syphilis von 15 Patienten 2 positiv.

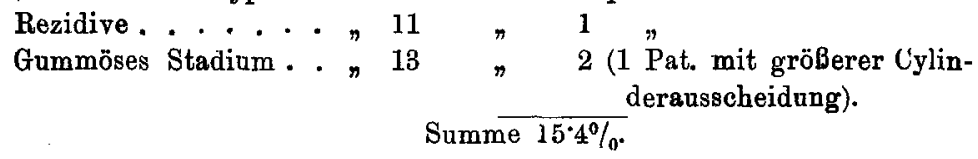

2. Reibe.

1. Ausbruch der Syphilis von 46 Patienten 6 positiv.

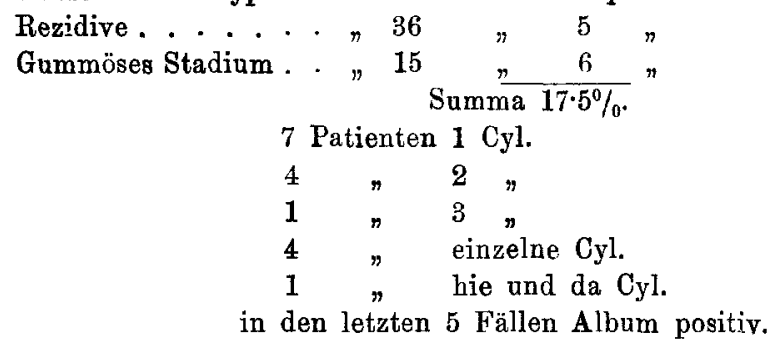

Auffallend ist es, daß bei Welanders Untersuchungen in beiden Reihen die gummösen Patienten in größerem Prozentsatz Cylinder in der Schwankung phys. Breite aufwiesen. Die Untersuchungen wären nach dieser Richtung hin fortzusetzen.

Aus Welanders und meinen Beobachtungen scheint hervorzugehen, daß eine während der Eruptionsstadien der Syphilis auftretende Cylindrurie, wenn sie überhaupt existiert, zu den großen Seltenheiten gehören muß.

Beobachtungen während der Hg-Kur: Meine Beobachtungen erstrecken sich auf 31 Männer, von denen 20 vor oder knapp nach Beginn der Inunktionskar und fortlaufend während derselben untersucht wurden, während von 11 Patienten zur Kontrolle ein oder mehrere Stichproben hintereinander während der Behandlung entnommen wurden.

Von 6 Frauen wurden zwei fortlaufend, von 4 nur einzelne Sedimentproben der mikroskopischen Besichtigung unterworfen.

Die Inunktionskur bestand in der auf der Klinik üblichen Weise aus Cyclen (6 Einreibungen à $4 g$ Unquentum cinereum), zwischen welche nach der 6. Einreibung ein Pausetag mit Vollbad eingeschaltet wurde. Außerdem kam graues Pflaster und 
weiße Präzipitatsalbe lokal zur Anwendung, ersteres nur in zwei Fällen (Nr. 18, Nr. 16) in größerer Ausdehnung.

Am besten dürften die während der Hg-Kur auftretenden Sedimentveränderungen durch die kurze Wiedergabe einer Krankengeschichte illustriert werden, während ich bezüglich der übrigen auf die der Arbeit angefügten Protokolle verweise.

Nr. 15. St., 25jähr. lediger Flößer, aufgenommen am 2./XII. 1905. Bisher nicht behandelt, erste Eruption. Lues cutanea mac. papulosa. Phimosis inflammata, Balanoposthitis, Sclerosis initialis, Scleradenitis univ. praecipue inguinalis. Starker Alkoholiker, Branntwein, Bier.

Therapie: Inunktionen, graues Pflaster, Kupferspülung.

5./XII. $\left.{ }^{1}\right)$ Spezifisches Gewicht 1030, Eiweiß negativ, Probe (1-3), Schleimfäden, massenhaft Leukocyten, wenig Epithelien, keine cylindrischen Gebilde, Beginn der Schmierkur.

7./XII. Spiegler, positiv, die Hellersche und Ferocyankaliessig. säureproben negativ. Im Sediment: Status idem.

11./XIl. E. $(1-3)$ negativ, spezifisches Gewicht 1025, Leukocyten mehr als Epith. sehr reichlich. In mehreren Gesichtsfeldern die später des Genaueren zu besprechenden cylindrischen Gebilde. Ca. 1-4 im Gesichtsfeld. 1. Tour beendigt.

14. XII. St. idem., E. (1-3) $\theta$, spezifisches Gewicht 1028.

16./XII. In vielen Gesichtsfeldern mehrere cylindrische Gebilde, Epithelien und Leukocyten wie gewöhnlich, spezifisches Gewicht 1025. E. $(1-3) \theta$.

18./XII. In fast jedem Gesichtsfeld sehr reichlich cylindrische Gebilde, typischer Befund. 2. Tour beendet, spez. Gewicht 1024. E. (1-3) $\theta$.

19./XII. St. idem., E $\theta$, spezifisches Gewioht 10.20.

22./XII. E (1-3) $\theta$, spezifisches Gewicht 1022, große Menge cylindrischer Gebilde, typischer Befund.

23./XII. Nach 16 Inunktionen wird Patient gebessert entlassen.

Man sieht also schon nach der sechsten Einreibung, nachdem die vorherige zweimalige Untersuchung, die wegen der vorhandenen Phimose und dem dadurch bedingten reichlichen Auftreten von Leukocyten und Epithelien im Sediment das Aufsuchen der "physiologisch" ja so häufig vorkommenden spärlichen hyalinen Cylinder erschwert haben mochte, negativ ausgefallen war, reichliche homogene, cylindrische Gebilde auftreten und im weiteren Verlaufe bis zum Abbruch der Kur (nach 16 Inunktionen) an Menge zunehmen. Mit Ausnahme des zweiten Tages, an den bloß die mit dem Spieglerschen

1) Der Harn stammt von der dem Tagesdatum vorhergehenden Nacht. 
Reagens ausgeführte Probe positiv war, während die übrigen Eiweilpproben ein negatives Resultat aufweisen, waren die Reaktionen auf Albumen stets negativ. Das spezifische Gewicht des Harnes sank während der Behandlung mit $\mathrm{Hg}$ (vermehrte Diurese). Auf die Natur der cylindrischen Gebilde komme ich später ausfübrlich zurïck.

Um einen Maßstab für die Bestimmung der quantitativen Verhältnisse der cylindrischen Gebilde zu geben, babe ich folgende Wertung gewählt, mir wohl bewußt, daß diese approximative Schätzung Fehler in sich einschließt.

3-6 cylindrische Gebilde im Präparat = zäblbar.

Bis ca. 12 Cylinder im Präparat = dentlich vermehrt.

$1-3$ Cylinder in vielen Gesichtsfeldern $=$ reichlich.

3-10 " " " = sehr reichlich.

darüber hinaus eine große Menge.

Bei dem Umstand, dabj verschiedene Gesichtsfelder desselben Präparates unter anstoßenden Gruppenbezeichnungen eingereiht werden mußten, konnte natürlich der allgemeine Eindruck zur Klassifikation entscheiden, doch machte im ganzen die Rubrizierung der Befunde keine Schwierigkeiten.

Die mikroskopische Schätzung wurde stets mit dem III. Okular und dem Objektiv Nr. 3 (Reichert) vorgenommen (die genauere Untersuchung der Gebilde natürlich mit stärkeren Vergrößerungen. Obj. Nr. 6 und Nr. 8).

Ich möchte nicht unterlassen daraut aufmerksam zu machen, daß es sich bei reichlichem Schleimgehalt des Sediments (gallertartiger Bodensatz) empfiehlt, dem Sedimenttropfen am Objektträger einen Tropfen phys. Kochsalzlösung zuzusetzen, da das dichte Gewirr der Schleimfäden die cylindrischen Gebilde verdecken kann. Es gelang in manchen Fällen, sie durch die Verdünnung sichtbar zu machen, während sie ohne dieselbe nicht zum Vorschein kamen.

Als Resultat dieser Untersuchungen ergibt sich: Bei 31 Männern und 6 Frauen war mit A usnahme eines einzigen Falles ein mehr oder minder reichliches Auftreten ron cylindrischen Gebilden im Harnsediment zu konstatieren, wobei die Quantität derselben meist proportional der einverleibten $\mathrm{Hg}-\mathrm{M}$ enge zunahm.

Ich muß es späteren Bearbeitern dieser Frage überlassen, die einzelnen Faktoren, die auf die Ausscheidung der cylindri- 
schen Gebilde einen Einfluß haben können, an einem größeren Material zu analysieren und mu mich begnügen, an meiner kleinen Untersuchungsreihe einzelne in Betracht kommenden Gesichtspunkte zu erörtern.

Von 31 Männern standen $4 \mathrm{im}$ Alter von 17-20 Jahren.

\begin{tabular}{|c|c|c|c|c|c|}
\hline & 18 & $n$ & $n$ & $n$ & $20-30$ \\
\hline & 6 & $\because$ & $"$ & $"$ & $30-40$ \\
\hline & 2 & $\eta$ & $"$ & $\pi$ & $40-50$ \\
\hline & 1 & $"$ & $"$ & $n$ & von 51 \\
\hline Von den Frauen standen & 5 & $"$ & " & $n$ & $18-20$ \\
\hline & 1 & $\pi$ & $\pi$ & $"$ & 24 \\
\hline
\end{tabular}

Der einzige negative Fall [Mann] stand im Alter von 25 Jahren.

Welander zieht aus T'ab. VI seiner Arbeit den Schluh, daß mit steigendem Alter der Prozentsatz derjenigen Patienten, die eine Menge von Cylindern ausscheiden, zunimmt, so daß unter 40 Jahren nur $27 \%$, über 40 Jahre $70 \%$ einen hohen Cylindergehalt aufweisen. Sehr interessant wäre es nun an Säuglingen und Kindern die Versuche fortzusetzen. Setzen die Harnwege so jugendlichen Alters dem Hg-Einfluß einen größeren Widerstand entgegen, als die durch Alkohol und starke Muskelarbeit weniger resistent gewordenen Organe des Erwachsenen? In einer der Welanderschen Mitteilungen (dieses Archiv Bd. XLVI) konnte ich die Angabe finden, daß ein $2^{\mathbf{1}} / \mathrm{j}$ jähriges Kind nach 22tägigem Gebrauch von We land erschen Säckchen à $2 g$ reichlich Cylinder im Harn darbot, dem sich am 30. Tag Albumen zugesellte.

Immerhin lohnt es sich diese Frage an einem größeren Material zu entscheiden.

Zur Orientierung über den Beginn der Ausscheidung der cylindrischen Gebilde möge folgende Tabelle dienen: 
Während der ersten Tour waren die cyl. Gebilde

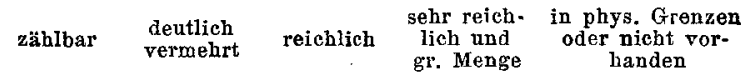

$$
\begin{aligned}
& \left.\begin{array}{rr}
4^{3} \\
6^{3} \\
11^{3}
\end{array}\right\} 3 \quad 1^{5}\left\{\begin{array}{rr}
12^{3} \\
1 & 9^{4} \\
8^{5} \\
13^{5}
\end{array}\right\} 4 \begin{array}{c}
12+2 \\
4
\end{array} \quad \begin{array}{c}
\text { (die mit negativem } \\
\text { Befund naeh der } \\
\text { 1. Tour das Spital } \\
\text { verlioBen) }
\end{array}
\end{aligned}
$$

Während der zweiten Tour

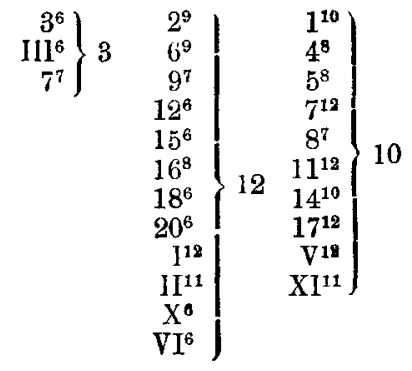

\}$^{19^{12}} 1$

Während der dritten Tour

$$
V^{16}\left\{\begin{array}{rr}
1 & 2^{16} \\
7^{18} \\
12^{18} \\
18^{14} \\
15^{16} \\
20^{18} \\
V^{16}
\end{array}\right\} 7
$$

Während der vierten Tour

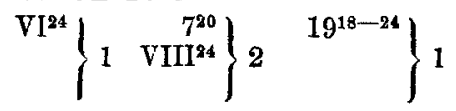

Während der fünften und sechsten Tour

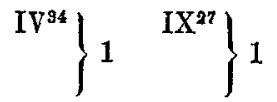

Die arabischen und lateinischen Grundzahlen bedeuten das $\mathrm{Nr}$. des Patienten in meinen Protokollen, die ersteren die fortlanfend untersuchten, die letzteren die Stichproben. Die Potenzzablen bedeuten die Zahl der Einreibungen, nach welcher die Ausseheidnng der cylindrischen Gebilde konstatiert wurde.

1) Wohl wurden bei dem Falle Pat. Nr. 19 nach viermaligem negativen Untersuchungsresultate am Beginne der 3. Tour reichliche Ausscheidung von cylindrischen Gebilden notiert, doch dürfte, da auch 
Da die Entnahme von Sedimenten nach gewissen Zeiträumen erfolgte, so ist nicht immer der Termin des ersten Auftretens der "Cylindrurie" getroffen worden, so daß er bei manchen Fällen um einige Tage zurückliegen kann. Immerhin gewährt die Tabelle doch ein anschauliches Bild dieses Symptoms.

Man sieht, daß schon nach der dritten Einreibung in drei Fällen die cylindrischen Gebilde zählbar, in einem Falle nach der 5. Inunktion deutlich vermehrt und in 4 Fällen nach der 3., 4., 5. Einreibung reichlich aufgetreten sind. $\mathrm{Ob}$ bei letzteren Patienten 12, 9, 8 die begleitende Oxalurie eine Rolle spielt, bleibt vorläufig unentschieden, ebenso ob bei Pt. Nr. 13 eine der Hg-Kur knapp vorhergegangene fieberhafte Angina eine Disposition geschaffen hat.

Von der 8. Inunktion ab ist die Ausscheidung bei allen Fällen mit Ausnahme eines zum mindesten reichlich, doch weisen schon 4 Fälle nach der 6. Hinreibung den gleichen Gehalt auf. Sehr reichlich sind die Formelemente einmal nach der 7., zweimal nach der 8, zweimal nach der 10., zweimal nach der 12. Einreibung notiert.

Während also im Verlaufe der ersten Tour die Zahl der negativen Fälle prävaliert, vermindert sich dieselbe am Anfang der zweiten Tour und schon nacb der 8. Einreibung scheidet der größte Teil der Behandelten eine reichliche Zahl von cylindrischen Elementen aus, die bis zu der 12. Finreibung noch eine bedeutende Steigerung erfäbrt.

Es sei hier gleich bemerkt, daß sich die Patienten bis auf eventuelle stomatitische Beschwerden wohl befanden, und daß auf die Regelmäßigkeit der Stuhlentleerung mit Rücksicht auf die Koblerschen Befunde geachtet wurde.

Über den Beginn und Verlauf der in Rede stehenden Affektion bei der Inunktionskur existieren bisher keine systematischen Untersuchungen. (Siehe das Nachwort.)

Welander hat bei den von ihm verordneten Überstreichungen $(6.0$ pro dosi) in $57 \%$ der Patienten, die

die vier späteren Sedimentproben vollkommen negativ ausfielen (trotz genauester Untersuchung), eine Verwechslung des Spitzglases vorgelegen haben, trotzdem die Kontrolle meist von mir persönlich ausgeführt wurde. 
mehr als 30 Überstreichungen erhalten hatten, eine bedeutende Menge von Cylindern beobachtet, während diese Menge von Cylindern sich nur bei $16.6 \%$ von denjenigen gezeigt haben, denen weniger als 30 Überstreichungen gegeben worden waren.

Eine Analogie mit unseren Beobachtungen zeigen die ron Welander der Injektionstherapie mit unlöslichen $\mathrm{Hg}$-Salzen unterworfenen Kranken, von denen nach 5 Injektionen $0 \%$, nach 6 Injektionen $33 \%$ und nach 7 Injektionen $38.5 \%$ eine bedeutende Menge von Cylindern ausschieden.

Es ergibt sich also aus $W$ e $I$ a n $d$ e $r$ s Resultaten und den meinigen (Inunktionstherapie), da $B$ die $M e n g e$ des applizierten $H g$ einen Einfluß auf die Größe der Ausscheidung der cylindrischen Gebilde hat.

Auffallend ist es uun, daß schon so geringe $\mathrm{Hg}$-Mengen, wie sie bei der Inunktionskur nach den grundlegenden Versuchen von $W$ internitz und $B$ ürgi ausgeschieden werden, eine Reizung in den Harnwegen hervorbringen können. Mit der Zunahme der Inunktionen und der dadurch bedingten steigenden Menge von eliminiertem $\mathrm{Hg}$ geht eine Vermehrung der cylindrischen Gebilde parallel im Harne einher.

Die Durchschnittswerte der Hg-Elimination nach Inunktionen mit täglich $4 g$ grauer Salbe in 6tägigem Turnus sind nach Winternitz folgende: Im Liter Harn wurde

nach 6 Einreibungen: 0.0001 Quecksilber

$\begin{array}{lllll}" & 12 & \# & 0.00055 & \\ & 18 & " & 0.00049 & 0 \\ & 21 & \# & 0.001 & \end{array}$

gefunden. Diese Zahlen betreffen Individuen mit pathologisch unveränderter Haut.

Die Ausseheidung des $\mathrm{Hg}$ beginnt bereits 1-2 Tage nach der ersten Inunktion.

Doch spielen bei der Wirkung des $\mathrm{Hg}$ auf die einzelnen Organe des Körpers außer der Größe der Dosis sicher individuelle Verhältnisse eine bedeutende Rolle. Klinik (W el and e r und Tierexperiment (Elbe, Klemperer, Karvonen etc.) stimmen in diesem Punkte überein, und so dienen folgende zwei Fälle durch ibre Gegenüberstellung zur Veranschaulichung der bekannten Tatsache: Fall Nr. 7. Der schwächliche, anämische Patient mit beiderseitiger Spitzenaffektion und reich- 
lichem papulösem Exanthem als erster Eruption zeigt erst nach der 7. Einreibung bloß deutlich vermehrten "Cylinder"gehalt des Harns, der aber schon nach der 12. Einreibung sehr reichlich wird. Im Gegensatz dazu Fall 20, ein kräftiger Mensch, mit macul. papulösem Exanthem, bei dem schon nach der 6. Inunktion reichliche Cylinder sich im Sedimente finden.

Was nun den einzigen negativen Fall betrifft, so erhob sich die Frage, ob $\mathrm{Hg}$ hier durch den Harn in entsprechender Menge ausgeschieden werde. Nach der 20. Einreibung wurde der Harn im Institute für physiologische Chemie auf $\mathrm{Hg}$ qualitativ untersucht. Herrn Prof. v. Zeynek, dem Vorstand des Institutes, und seinem Assistenten Lippich sage ich für die Anleitung bzw. freundliche Unterstützung bei der Analyse meinen besten Dank.

In oa. $1000 \mathrm{~cm}^{3}$ mit Salzsäure versetzten Harnes werden 2 Platinelektroden getaucht und durch 24 Stunden unter mehrfachem Umrühren ein Strom von 4 Yolt Spannung durchgeleitet. Die Elektroden wurden mit destill. Wasser, Alkohol und Äther gewaschen und kommen dann in eine Röhre von Hartglas. $\mathrm{CuO}$ und $\mathrm{CaO}$ werden vorgelegt und nachdem das $\mathrm{CuO}$ zur schwachen Rotglut erhitzt wurde, die Platinelektroden erwärmt, das $\mathrm{Hg}$ in der abgesprengten Kapillarröhre durch Joddämpfe in gelbes Quecksilberjodid übergeführt, nach Absaugung des überschüssigen Jods durch Reiben mit einem kapillar ausgezogenen Glaßstab in die rote Modifikation umgewandelt. Hg war schătzungsweise reich. lich vorhanden.

Der Verlauf der „Cylindrurie " ist, wie schon früher angedeutet wurde, individuell variierend. Nicht immer kommt es zur bedeutenden Steigerung der "Cylinder" ausscheidung. So hat im Fall 4. die Ausscheidung der Formelemente rom Beginne der 2. Tour bis zum Ende der 4. Tour nur um ein geringes zugenommen. Auch in Falle IV, der erst während der 34. Inunktion untersucht wurde, war die Menge der cylindrischen Gebilde nur reichlich. Hat die „Cylindroidurie ( ${ }^{1}$ ) eine best. Höhe erreicht, so verbleibt sie auf derselben noch einige Zeit nach Abschluß der Behandlung (nicht protokolliert).

Über die Dauer der Cylindrurie nach Beendigung der Kur kann ich nur dürftige Angaben machen. Bei einem, ca. 50j. Manne waren 8 Tage nach dem Abbruch einer 3

1) Solange nicht in die Morphologie der Gebilde Klarheit gebracht ist, möchte ich mit der endgültigen Bezeichnung dieses Symptoms noch zurückhalten. 
Touren umfassenden Schmierkur noch sehr reichliche cylindrische Gebilde nachweisbar. Im Falle Nr. 16, der nach der 8. Einreibung infolge einer Stomatitis die $\mathrm{Hg}-\mathrm{Kur}$ abbrechen mußte (Cyl. in gr. Menge vorhanden), fanden sich $4 \mathrm{~W}$ och e $\mathrm{n}$ später noch „reichlich“ cyl. Gebilde im Sediment.

Die Beobachtung scheint mir darum von Interesse, weil schon eine relativ kleine Hg-Menge langdauernde Nachwirkung hinterlassen kann. Im Falle 17 war der Anamnese nach eine aus 3 Touren bestehende Inunktionskur 6 Wochen vor der Aufnahme des Patienten vorausgegangen. Cylindrische Gebilde waren (mit Ausnahme eines hyal. Cylinders nach der 3. Einreibung) nicht zu entdecken. Nach Welander ist in der Regel der Cylindergehalt 4-6 Wochen nach Schluß der Behandlung verschwunden. Doch beobachtete er einen Fall, wo eine bedeutende Cylindrurie 10 Wochen nach beendigter Behandlung vorhanden war. - "Wie lange es währt, bis der Cylindergehalt verschwunden ist, beruht wahrscheinlich auf der größeren oder geringeren Menge Cylinder, die sich bei Schluß der Behandlung finden, sowie auf der größeren oder geringeren Menge $\mathrm{Hg}$, das bei der Behandlung angewendet worden ist."

Mit Rücksicht auf die Untersuchungen Bürgis über die lange Remanenz des $\mathrm{Hg}$ wären Untersuchungen mit der von mir verwendeten Methode über die Dauer der "Cylindrurie" nach Abbruch der Kur, denen sich allerdings Schwierigkeiten in den Weg stellen, gewiß erwünscht.

Hat die einmalige oder mehrmalige Vorbehandlung mit $\mathrm{Hg}$ einen Einfluß auf die „Cylindrurie?" Welander warnt, die mit der Vermehrung der Kuren steigenden Prozentzahlen von Ausscheidung großer Mengen von Cylindern der Vorbehandlung zuzuschreiben, sondern führt sie auf die für schwere Formen von Syphilis notwendige intensive Hg-Anwendung zurück. Von 19 unbehandelten Fällen zeigte nur einer eine bloße Vermehrung der cyl. Gebilde, doch trat er schon nach der ersten Tour aus der Klinik, von den 12 vorbehandelten Fällen war bei 2 Patienten der Cylindergehalt als bloß vermehrt notiert, und auch diese verließen schon nach Beendigung der ersten Tour das Krankenhaus. Sonst waren die Cylinder in allen Fällen mindestens "reichlich" vorhanden. Der einzige 
negative Fall war dreimal vorbehandelt. $[1.3 \mathrm{mal} 4$ Touren und 2 Inj., 2. nach $11 / 2 \mathrm{Jahr} 5$ Touren, 3. nach 5 Monaten 5 Welandersche Säckchen.]

$\mathrm{Da}$ b die Affektion des uropoetischen Systems mit der $\mathrm{Hg}$ Stomatitis in Beziehung steht, wird von Welander verneint und auch ich glaube, daß die folgenden zwei Krankengeschichteu die Beibringung statistischer Daten entbehrlich macht.

N., 16jährige geheime Prostituierte, bekam nach 3 Inunktionen eine heftige Stomatitis, ohne daß sich diese Gebilde auch bei später angewandten $5 \%$ Sublimatinjektionen zeigten. Im Kontrast dazu steht Fall VIII, der nach der 4. Tour eine mächtige Cylindrurie aufwies und trotz $181 \%$ Sublimatinjektionen, die sich der Inunktionskur anschlossen, niemals über die geringsten stomatitischen Beschwerden klagte.

Die Frage, ob unter die zur Ausscheidung der cyl. Gebilde disponierenden Momente auch der Alkoholismus zu rechnen ist, wird von W elander nicht berührt. Meine Zahlen sind zu klein, um eine Beantwortung derselben zu ermöglichen. Doch möchte ich darauf hinweisen, daß der vollständig vegative Fall (Nr. 19) und Fall 6 und 7 mit bloß reichlicher "Cylindrurie $^{*}$ trotz ausgiebiger $\mathrm{Hg}$-Behandlung niichterne Menschen waren, die jedweden AlkoholexzeB in Abrede stellten. Damit soll natürlich nicht behauptet werden, daß Temperenzler nicht eine mächtige Cylindrurie unter $\mathrm{Hg}$-Einfluß bekommen können. Doch müssen mit Bezug auf Glasers' Fxperimente, nach denen Alkoholexzesse das Auftreten einer größeren Zahl von Cylindern im Harn zur Folge haben, Versuche nach der angegebenen Richtung hin, von Interesse erscheinen.

Die Untersuchungen an Weibern konnten, da sie mit Schwierigkeiten verbunden sind (Katheterismus des Morgenharns, übler Wille der Prostituierten! etc.), nur in beschränkter Zahl vorgenommen werden.

2 Fällen mit 24 bzw. 19 . Einreibungen und reichlichem "Cylinder"gehalt, einem Fall mit 9 Inunktionen und deutlicher Vermehrung der cyl. Gebilde reihen sich 2 Patientinnen mit einer gr. Menge von „Cylindern" nach 22 bzw. 25 Einreibungen an. Sehr wichtig erscheint es mir die Untersuchungen auch auf

1) Glasers Experimente, die mit frisch gelassenem. Harn angestellt siud, wären mit der. Modifikation der Nubeculazentrifugierung zu wiederholen. 
luetische Schwangere auszudehnen mit Berücksichtigung des Umstandes, ob die Schwangerschaftsniere und die von Fischer während der Gravidität beobachtete Cylindrurie ohne Eiweißausscheidung nicht eine exakte Kontrolle des Sediments erfordert.

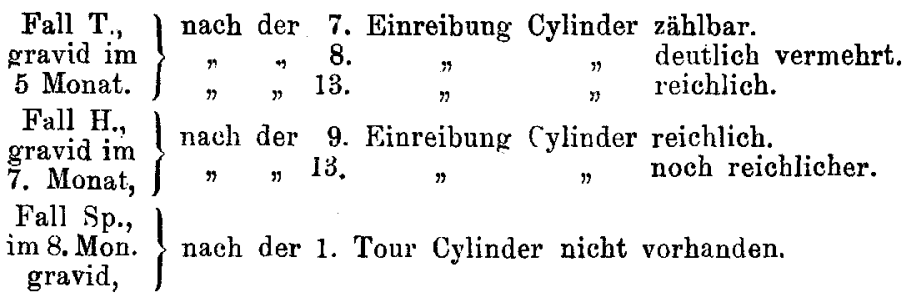

Aus diesen Fällen ist ein abnormes Verhalten nicht zu ersehen. Doch ist die weitere Prüfung gewiß eine Notwendigkeit. Erwähnt sei hier die Mitteilung Brugschs, die Niere der gesunden Frau sei gegen Salizyl weniger resistent als die des Mannes. Die Wirkung des Quecksilbers auf den Harnapparat nach dieser Richtung hin zu studieren, wird des Interesses nicht entbehren.

Mit Einreibungen (1 Tour) und nachfolgenden 5\% Sublimatinjektionen ( $1 / 2$ Spritze jeden 5. Tag) wurden 4 Fälle behandelt -2 waren negativ.

Über die Sedimentveränderungen narh Injektionsbehandlung werde ich demnächst berichten.

Ich teile bloß die Krankengeschichte eines Mannes mit, der mit $1 \%$ Sublimatinjektionen behandelt wurde, die auch noch in anderer Hinsicht bemerkenswert erscheint.

S., 21j. lediger Arbeiter, aufgen. am 6./XI. auf die chir. Klinik wegen Kontusion der Niere. Ein am 13./XI. bemerktes Exanthem veranlaßt seine Transferierung anf die dermatologische Klinik.

Inf. im März 1905. Im April eine Tour geschmiert.

Diagnose: L. cutanea mac. papulosa. Pap. seborrh. capill. Papulae in regressu glandis. Papulae elevatae partim exulcer. ad anum. Sclerosis sanata. Scleradenitis univ. praecipue inguin. et cubit. Gonorrhoea subacuta. Harnbefund am 13./XI. Vormittagsharn. E +. sp. G. 1028. Hyaline Cylinder mit Leukocyten, mit roten Blutkörp. besetzte Cyl. Granul. Cylinder.

14/XI. Status idem.

18./XI. 1. Inunktion.

19. / Xl. Inunktion ausgesetzt. 
20./XI. 1. Injektion. Hyalin, granul. Cyl. 6-8 im Präparat.

21./XI. 4-6 hyal. u. inkrust, Cyl. im Präp. Rote Blutkörperchenschatten. Ferocy. Essigs. +? Heller +? Spiegler +. sp. G. 1024.

28./XI. Nach der 5. Inj.: Ferocy. Essigs. schwach +. Heller +? Spiegler +. sp. G. 1020. Sehr reichl. cyl. Gebilde, wie bei den übrigen mit Hg behandelten Fällen spärliche granul. und mit Leukocyten besetzte Cyl. Lange hyaline Cyl. Einige Schleimcylindroide.

4./XII. Nach der 8. Injektion: sp. G. 1022. Eiweiß +. 4-8 cyl. Gebilde (für $\mathrm{Hg}$ typisch) im Gesichtsfeld. Ke ine granulier ten Cylinder, nur noch einige dicke, hyaline, kleine Cylinder.

12./XII. Fl. essigs. +. Heller +? Spiegler +. 1023. Nach 12. Inj. Ein feingranulierter Cylinder, sonst der typische Befund nach Hg-Einreibung.

16./XII. St. idem. Ke in granl. Cylinder. Eiweiß positiv. Sp. G. 1020. Gebessert entlassen nach 13 Injektionen. Im Sediment sehr reichliche cyl. Gebilde.

Es sind also hier während der Quecksilberbehandlung die Blut-, Leukocyten- und Epithelcylinder, ebenso die granulierten Cylinder aus dem Sedimente verschwunden, um dem nach Merkurdarreichung typischen Befunde Platz zu machen. Demnach ist die Vorstellung gestattet, daß unter der Hygiene des Spitalsaufenthaltes (Bettruhe, Vermeidung von Alkohol) eine Besserung der traumatischen Nephritis statt hatte, unbehindert dadurch, daß das Quecksilber seinen Einfluß auf die Harnwege geltend machte. Dex Eiweißgehalt des Harnes hielt sich stets in so mäßigen Grenzen, daß von einer quantitativen Bestimmung Abstand genommen werden konnte. Man muß mit Welander ibereinstimmen, der in einer vorhandenen Nephritis keine Kontraindikation für die Quecksilberbehandlung einer interkurierenden Lues findet, vorausgesetzt, daß der Harn und das Sediment, dessen Veränderung während der $\mathrm{Hg}$-Behandlung bekannt sein muß, ständig kontrolliert werden.

Zum Schlusse der Reihe der prädisponierenden Momente möchte ich noch die Oxalurie erwähnen. Es fällt auf, daß unter 4 Fällen mit reichlicher Ausscheidung von Cylindern während der ersten Tour bei drei Patienten, Nr. $\left.12(3), \mathrm{Nr} .9(4), \mathrm{Nr} .8(5)^{\mathbf{1}}\right)$, Oxalsäurekrystalle im Sedimente vorhanden waren.

Fall Nr. 12. Nach der 2. Einreibung in jedem Präp. 2 hyal. Cylinder. Nach der 3. Einreibung Oxalurie aufgetreten mit reichl. Aus-

1) Die in Klammer befindlichen Zahlen bedeuten die Zahl der Einreibungen, nach welchen Cylindrurie beobachtet wurde. 
scheidung cyl. Gebilde. Zwei granul. nnd ein Epithelcylinder. Nach der 6. Finreibung in mebreren Gesichtsfeldern ein cyl. Gebilde:

Fall Nr. 9. Nach der 1. Einreibung 2 hyal. Cylinder mit cylindroiden Anhängen, massenhaft Oxalsäurekrist. in jedem Gesichtsfeld. Nach der 2. Einreibung ein hyal. Cyl. mit einem Leukocyten, spärliche Oxalsäurekrist. Nach der 4. Einreibung reichl. cyl. Gebilde, Oxalsäure negativ.

Fall Nr. 8. Nach der 3. Einreibung Oxalsäure reichlich, ein feingranul. Cylinder. Nach der 5. Einreibung Oxalsäure $\theta$. Cylinder reichlich.

Fall S., ${ }^{1}$ ) Mann. 13./XII. $1 / 2$ Spritze $5 \%$ Sublimat.

14./XII. Starke Oxalurie, Schleimfäden, Leukocyten, Lpithel reichlich. Spiegler pos. Fe. Essigs. und Heller negativ.

15./XII. Spiegler pos. Die beiden anderen Proben negativ, sp. G. 1034. 3 kleine homogene cyl. Gebilde, ein br. halbgranul. Cylinder.

18./XII. Oxalsäure $\theta$. Cyl. $\varnothing$. Sp. +. 1034 .

20./XII. 2 homog. Cyl. in jedem Präparat. Sp. + .

Ich muß es unentschieden lassen, ob die Oxalsäure an sich bereits "Cylindrurie" hervorrufen kann oder ob sie bloß prädisponierend dem $\mathrm{Hg}$ einen Locus minoris resistentiae darbietet. Auch kann jch ein bloß zufälliges Zusammentreffen nicht ausschließen. Anzufügen wäre bier eine Beobachtung Töröks, der nach reichlicher Harnsäureausscheidung das Auftreten von renalen Cylindroiden im Sediment erwähnt.

\section{Die Morphologie und Reaktionen der Gebilde.}

Die cylindrischen Elemente stehen den von Török als renale Cylindroide beschriebenen Gebilden sehr nahe. Beror ich sie mit denselben identifizieren kann, bedarf es aber experimenteller Untersuchungen und zufälliger Autopsien von mit $\mathrm{Hg}$ behandelten Individuen. Untersuchungen an Hunden sind bereits in Angriff genommen.

Es handelt sich um homogene, meist matt glänzende Gebilde; sie sind bald ovale, an beiden oder nur an einem Ende abgestumpfte Gebilde, häufig fadenförmig, bandartig, keulenförmig, von verschiedenster Länge. Sie haben im Gegensatz zu den erst spät auftretenden, gut modellierten, walzen-

1) Verließ nach der 1. Injektion die Klinik, ist in die Protokolle nicht aufgenommen. 
förmigen hyalinen Cylindern, die den zum Vergleich herangezogenen bei parenchymatöser Nephritis ausgeschiedenen gleichen, ein schlappes matsches Aussehen, als ob es sich nicht um solide Ausfüllungen eines Röhrenlumens handelte, sondern am leicht biegsame Schläuche oder platte Bänder. Dafür spricht auch die häufig beobachtete Quer- und Längsfältelung derselben, die in einer feinen Quer- und Längsstreifung zum Ausdruck kommt. Die der Quere nach zusammengeschobenen Cylinder können eine Entstehung aus hyalin degenerierten Zellen vortäuschen, doch wird eine genaue mikroskopische Untersuchung vor diesem Irrtum bewahren. Einige besitzen an dem cylinderförmigen Stamm ein spiralig korkzieherartig oder schraubenförmig gewundenes Ende. Messungen ergaben für die kleineren Gebilde $16 \mu$ Breite, $24 \mu$ Länge, $16 \mu$ Breite, $48 \mu$ Länge, $8 \mu$ Breite und $128 \mu$ Länge etc.

Bloß in 2 Fällen konnte ich den von Lüthje bei der Salizylnephritis abgebildeten hyalinen Kugeln ähnliche Gebilde finden. Die Färbung geschah durch Zusatz eines Tropfens der Farblösung zu einem Sedimenttropfen am Objektträger.

Die saueren Farbstoffe Eosin, Lugol, saueres Fuchsin brachten die Gebilde nicht deutlich zum Vorschein. Sie nehmen die Farbe nur in geringem Maße an.

Am besten bewährte sich das polychrome Methylenblau und ihm zunächst Methylenazur, das von Kraus zur Färbung der Hyphomyceten angegeben wurde. Mit polych. Methylenblau färben sie sich bald in einem rosa, bald in einem violetten Ton und kann ich die beiden Farbstoffe zur Aufsuchung der Gebilde aufs wärmste empfehlen. Auffallend ist es, daß die Gebilde an einem Ende stärker gefärbt erscheinen. Möglicherweise hängt dies mit der verschiedenen Dicke derselben zusammen.

Das Pappenheimsche Methylgrün-Pyroningemisch (im hängenden Tropfen) (S enator) gab mir keine günstigen Resultate. Mit Haematoxylin (Delafield) nehmen sie eine blaßviolette Färbung an.

Die Konservierung der Elemente für einige Tage wird durch Zusatz von einigen Thymolkristallen zum Sediment bewirkt, soll sie für längere Zeit bewerksteiligt werden, so empfiehlt sich 
eine $2 \%$ Formollösung mit vorherigem gründlichen Auswaschen mit phys. Kochsalzlösung. Der Versuch, das Sediment in Paraffin einzubetten, ähnlich den von Gabritschewsky für das Sputum empfohlenen Vorgang, gelang nicht, da der nach der Formolfixierung angewandte Alkohol die Elemente zur starken Schrumpfung brachte.

Im Wasserbade auf $65^{\circ}$ erwärmt lösen sich die Gebilde, ebenso in destill. Wasser bei Zimmertemperatur.

Normalnatronlauge und $50 \%$ Essigsäure haben die gleiche Wirkung.

Außer den beschriebenen cyl. Formelementen und hyalinen Cylindern wurden in 4 Fällen während der 1. Tour mit Epithelien und Leukocyten besetzte Cylinder beobachtet, einmal ein fein graul. Cyl. (Nr. 1, 14, im Falle 8, 9 war gleichzeitig Oxalurie zu konstatieren.) Im Verlaufe des zweiten Einreibungszyklus wurde einmal (Fall Nr.6) ein epithelialer Cyl. ausgeschieden.

Im Beginne und während des Verlaufs der Kur in einem Falle granulierte Cylinder (Oxalurie). Interessant ist ảer Befund bei Fall 2 (Insuff. et Stenosis valv. mitralis), wo nach der 16. Inunktion zugleich mit positivem Eiweißbefund "echte" hyaline Cylinder, die mit roten Blutkörperchen und Blutkörperchenschatten bedeckt sind, notiert wurden, am nächsten Tage aber sowohl das Eiweiß als auch die Erythrocytencyl. aus dem Harne verschwanden, während der typ. Befund an homogen cyl. Gebilden nach wie vor fortbestand. (Nach der 2. Inunktion einhalb mit Leuk. bes. Cyl.)

Nach Welander treten zuerst nur hyaline Cylinder auf, unter diesen später körnige, schließlich kann man auf den Cylindern hie und da ein oder mehrere Zellen unterscheiden, und mehr als einmal lassen sich zuletzt mehr oder weniger vollständige Epithelcylinder sehen. Scheinbar bedarf es längerer Kuren, um das letzterwähnte mit Albuminurie verbundene Stadium herbeizuführen. Denn in meinen Beobachtungen traten die granulierten Leukocyten oder Epithelcylinder nur vorübergehend und vereinzelt in Erscheinung.

Bezüglich der EiweiBausscheidung ist zu bemerken, daß sie nur in einem Falle deutlich auftrat, in einigen Fällen vor- 
übergehend die Spieglersche Reaktion allein oder Ferrocyankaliessigsäure und Spiegler bei negativer Hellerscher Ringprobe schwach positiv erschienen. Eine Albuminurie von längerer Dauer habe ich nie nachweisen können, wofür der Grund in der relativ kurzen Dauer der $\mathrm{Hg}$-Kur gesucht werden kann.

Daß der unerwartet große Prozentsatz „typischer Sedimentbefunde" nicht bloß einer Häufung von Zufällen zu verdanken ist, kann mit großer Wahrscheinlichkeit aus folgendem Versuch geschlossen werden. Zu Demonstrationszwecken bedurfte ich nach Abschluf der Arbeit mehrerer Sedimente. Die 4 einzigen Patienten (nicht protokolliert), die zu dieser Zeit sich auf der Klinik in dem entsprechenden Stadium der Hg-Kur befanden, wurden herangezogen und boten a us$\mathrm{nahmslos}$ den zu erwartenden Befund.

Nochmals sei zum Schlusse darauf hingewiesen, daß nur die Zentrifugierung der Nubecula ein sicheres Resultat ergibt. Von einem und demselben Harne (Anfángsstadium der Cylindroidurie) wurden in mehreren Fällen eine gewisse Menge sofort nach der Entleerung aus der Blase untersucht. Es fanden sich bloß spärliche Epithelien. Erst in der ausgeschleuderten Nubecula ließen sich die charakteristischen Gebilde nachweisen.

Es erschien mir nicht überflüssig zum Vergleiche der durch $\mathrm{Hg}$ hervorgebrachten Sedimentveränderungen mich mit der durch Salicyl. bewirkten Cylindrurie zu beschäftigen, zumal die Salizylpräparate sowohl bei externem als auch bei internem Gebrauche eine hervorragende Rolle im therapeutischen dermatologischen Inventar spielen. Bei äußerlicher Applikation (Einpackung der oberen und unteren Extremitäten bei zwei an Prurigo leidenden Knaben, Bedeckung von ausgedehnteren Flächen von mit chr. Ekzem. behafteten Patienten mit $5 \%$ Emplastrum salicylicum Pick) ließ sich trotz wochenlanger Anwendung Salizyl im Harne nicht nachweisen, es war daher eine Cylindrurie nicht zu erwarten und auch nicht beobachtet worden.

Es wurden nun 6 Patienten zwischen 15 und 40 Jahren (Männern) $5 \mathrm{~g}$ salizylsaueres Natron oder Aspirin in Dosen à $1 g$ über den Tag verteilt gereicht. Selbstverständlich wurde der Harn vor Beginn der Salizyleinnahme auf Anwesenheit von Eiweiß und Cylindern untersucht. Nur 
die beiden jugendlichen, an Prurigo leidenden Patienten zeigten intermittierend Albuminurie und von dieser oft unabhängige Ansscheidung von spärlichen Cylindern verschiedener Art. Es traten bloß wohlgeformte hyaline Cylinder bis zu 20 im Präparat oder hyaline und granulierte Epithelcylinder in wechselnder Menge auf, spärlich Schleimcylindroide, Epithelfetzen etc. $\left.{ }^{1}\right)$ Niemalsaber wurde ein dem nach Hg-Applikation von mir beschriebener gleicher Befund konstatiert.

Lü thj e sah nach Salizyldarreichung zuweilen „große Netze und Konglomerate von Cylindroiden, die einen mehr oder weniger großen Teil des Gesichtsfeldes verdeckten". Ich habe leider gerade keinen derartigen Befund zur Hand gehabt, dessen Vergleich mit den meinigen bei Hg-Behandlung erhobenen gewiß ron Interesse gewesen wäre. L üthje glaubt mit Wahrscheinlichkeit den renalen Ursprung der Cylindroide annehmen zu können, da er sie in durch Ureterenkathethrismus gewonnenen Harn nachweisen konnte. Jedenfalls ist ihr Entstehungsort oberhalb der Blase.

Eine Gefahr bringt selbst intensive, unter steter $K$ ontrolle des Harnes und Sedimentes vorgenommene $\mathrm{Hg}$-Behandlung nicht hervor und so konnte Welander bei genauen Untersuchungen seiner chronisch intermittierend behandelten Patienten niemals eine Schädigung der Nieren nachweisen.

Nicht bloß von wissenschaftlicher, sondern auch von praktischer Bedeutung wird es sein, bei den in industriellen Betrieben, mit Hg-Verwendung, beschäftigten Arbeitern die Veränderung der Harnsedimente durch Jahre zu studieren. Konnte doch Welander bei einer Warteperson, die sich in mit Quecksilberdämpfen geschwängerten Krankenzimmern längere Zeit beruflich aufhielt, eine Cylindrurie auftreten sehen.

Bereits im Feber vergangenen Jahres konnte ich die Resultate der Arbeit in einer Sitzung der biologischen Sektion des Lotos mitteilen. Mit der Publikation derselben hielt ich zurück, weil ich die Natur der cyl. Gebilde (renalen Cylindroide) erst genauer studieren wollte.

1) Ich verzichte auf die Wiedergabe der Protokolle, da sie nur die bekannten Befunde Lü thjes bestätigen. 
Da mir aber vor kurzem eine Arbeit von Klieneberger "Quecksilberschmierkuren und ihre Einwirkung auf die Harnorgane", Zeitschrift für klinische Medizin, LVIII. Band (pag. 481-498), 1906, zu Gesichte kam, die den gleichen Gegenstand behandelt und im Wesen zu gleichen Resultaten kam, so schreite ich zur Veröffentlichung. Klieneberger spricht von der Ausscheidung hyaliner Cylinder. Ich werde in der Fortsetzung der Arbeit, die ich gemeinsam mit Urbach unternehme, darauf zuriuckkommen, ob es gestattet ist, die fraglichen Gebilde (renale Cylindroide) mit echten hyalinen Cylindern zu identifizieren, und auch einige Punkte der Klien ebergerschen Arbeit einer ausführlichen Besprechung unterziehen.

Es ist mir eine angenehme Pflicht, Herrn Dr. Popper für seine freundliche Hilfe meinen besten Dank zu sagen.

\section{Protokolle der fortlaufend untersuchten Patienten (Männer):}

Nr. 1. T., 19jähr. Diener, aufgen. am 5./X. Reichl. Alkobolmengen blob an Sonntag. Sclerosis initialis, Scleradenitis univ. Iues cut. maculosa.

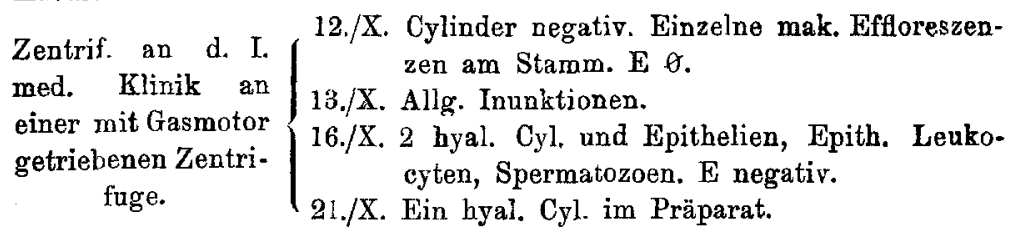
Elektr. Zentrif. 23./X. 20-30 Cyl. Geb. in jedem Gesichtsfeld. Sonst. Ep. und Leuk., entl. 24./X. mit Stomatitis.

Nr. 2. B., 22jähr. Bäckergehilfe, aufgen. 20./X. 1905 (trinkt nur Sonntags 6 Glas Bier. L. cut. maculo papulosa, tuberosa. Papulae labii inf., are palatoglossi. Pap. penis, scroti, ad anum. Selerad. univ. Leukoderraa colli. Insuff valv. mitr. cum stenosi.

Anfang 1904 Rheumatismus.

Im September 1904 Initialaffekt.

Im November bis Februar 1905 ein Ausschlag, der ohne BehandJung zurüekging.

24./X. Beginn d. Inunktionskur, spärliche hyal. Cyl., einzelne rote Blutkörperchen.

25./X. Status idem. Leukocyten, Epithelien reichlich.

26./X. Fin halb mit Leuk. besetzter Cyl. Sonst noch 1-2 hyal.

Cyl. in Präparat. 
30./X. 2. Tour begonnen. Exanthem im Rückgang. 4-6 byal. Cyl. in Präparat.

2./XI. Cyl. Gebilde deutlich vermehrt, ein inkrust. hyal. Cyl. sonst einzelne cyl. Gebilde. Status idem.

3./XI. Sehr reichl. cyl. Gebilde ca. 6-8 in jedem Gesichtsfeld.

10./XI. Sehr reichl. Cyl. Cyl. mit roten Blutschollen besetzt. E. + Sp. G. 1015. Leukacyten, wenig Epithelien.

11./XI. E. $\varnothing$. Keine Erythrocyten-Cylinder, einzelne rote Blatkörperchen und Blutk.-Schatten, sp. G. 1015. Sonst typ. Befund. Nach 3 Touren auf eigenes Verlangen entlassen.

Nr. 3. B., 27jähr. Kutscher, aufgen. 23./X., trinkt 5-6 Glas Bier täglich. Lues condylom. Pap. elev. madid. ad anum. Sclerosis ad anum, Scleradenitis univers.

Infektion Juli 1904.

Schmierte 8-9 Touren, zuletzt vor einem Jahr.

24./X. Erste Inunktion.

25./X. Cyl. $\theta$ (in 4 Präp. unters.) Leukocyten, rote, weiße Blutkörp. Platten- und Übergangsepithel.

26./X. St. jdem.

Eiweiß negativ 27./X. Im 5. Präparat ein hyal. Cyl.

Das im phys. Institut zentrif. Sediment nochmals. mit der klin. Zentrif, ausgeschleudert. Gleich im ersten Präparat ein hyal. Cyl.

28./X. Ein hyal. Cyl. in 2 Präparaten. Sonst St. idem.

30./X. Stomatitis. Schwellung der submax. Drüsen, 10 "Cylinder" im ersten Präp. 6 Cylinder im zweiten Präp. Sonst Status idem.

31./X. Auf eigenen Wunsch entlassen.

Nr. 4. F., 23jähr. Diener, aufgen. 25./X. Kein Alkoholiker. Pap. muc. oris. labiorum, tonsill. Papul, transform. penis. Pap. ad anum, Phimosis inflamm. Inf. August 1905. Vor 9 Wochen eine Tour geschmiert.

26./X. Erste Einreibung. In jedem Präparat 1-2 hyal. Cyl. Epithelien. Lueukoc. Rote Blutk. Harnsäurekristalle.

29./X. ca. 4-5 cyl. Geb. in Präparat.

30./X. c8. 4-5 cyl. Geb. in Präparat.

2./XI. 2. Tour begonnen.

4./XI. Nach der 8. Inunktion. In jedem Gesichtsfeld ca. 8-10 cyl. Geb., Leukocyten mehr als Epith.

6./XI, entlassen.

Nr. 5. J., 26jähr. Arbeiter, aufgen. 19./X., trinkt 6 Glas Bier täglich, Sonntags mehr. Ulous induratum, Lymphadenitis inguin. dextra supp. Sclerad. inquin cubit. Lymphangoitis dorsal. penis L. cut. mac. papulosa. Letzter Coitus vor 4 Wochen Inunktion.

26./X. macul. pap. Exanthem im Beginne am Stamm. Beginn dér 
27./X. Exanthem deutlicher. E. $\theta$. In 4 Präparaten keine Cylinder, das über Nacht unter Chloroform aufbew. Sediment wird am 28./X. neuerdings zentrifugiert. Im ersten Präparat: 2 hyal. Cyl. Plattenep. Leukocyten.

28./X. In einem Präparat ein hyal. Cyl.

29./X. Das gestrige Sediment nochmals auf d. kl. Zentrifuge zentrifugiert - keine Cylinder.

2./XI. 3-4 hyal. Cyl. Geb. im Präp. 2. Tour begonnen.

5./XI. Nach der 8. Einreibung (klinisch zentrifugiert) fast in jedem, Gesichtsfeld 6 Cyl. Geb. Schleim cylindroide, Leuk. Epith. E. $\varnothing$.

10./XI. 10-12 cyl. Geb. in fast jedem Gesichtsfeld. E $\theta$, sp. G. 1020. 11./XI. Typ. Befund, sp. G. 1025. E. $\theta$ entlassen.

Nr. 6. W., 45jähr. Öfner. Aufgenommen am 31./X. 4. Spitalsaufenthalt (Säufer). Pap. mucosae oris in regressu. Pap. mad. et exulc. penis, scroti, ad anum.

Inf. vor 3 Jahren. 14 Touren geschmiert. Zuletzt 4 Touren im April 1905.

2./XI. 2 kl. hyal. cyl. Geb, rote Blutkörp. spärlich. EpithelienLeukocyten. Schleim-Cylindroide.

3./XI. Nach d. 3. Einreibg. 4-6 hyal. oyl. Geb. Gebilde.

9./XI. Nach der 8. Einreibg. in vielen Gesichtsfeldern. 1-2 cyl.

10./XI. Nach d. 9. Einreibg. cyl. Geb, reichlicher als gestern. Plattenep. Übergep. Nierenep. (?) u. Leukocyten, ein epithelialer Cyl. E. $\theta$ sp. G. 1015.

14./XI. 1-2 cyl. Geb. fast in jedem Gesichtsfeld. wenig Epith. und Schleimfäden. Schleimcylindroide. Seit vorgestern Stomatitis. 2. Tour beendet.

16./XI. entlassen.

Nr. 7. K., 25jähr. Arbeiter, aufgen. 6./XI, (Kein Alkoholiker.) L. cutanea maculopapnl. Pap. seb. capillitii et faciei. Pap. ad marg. praep. Sclerosis sanata, Sclerad. univ. Catarrh. apicis pulm. lat. utriusque. (Gonorrhoe seit 9 Wochen.) Inf. August 1905. Seit 1 Woche Exanthem.

7./XI. Plattenep, die Leukocyten überwiegend, wenig Schleimfäden, keine Cylinder.

8./XI. 4-5 Schleimcylindroide im Präp., in drei Präp. keine cyl. Geb., Plattenep. und Ưbergangsep.

11./XI. St. idem. E. $\theta 1025$.

14./XI. Nach der ersten Tour Schleim-Cylindroide ziemlich reichlich. Cyl. Gebilde 6-8 in mehreren Präparaten. E. $\theta$.

20./XI. Nach der 2. Tour. In vielen Gesichtsfeldern bis 6 cyl. Geb. Epithelrasen, Lenkocyten. sp. Gew. 1015. E. $\theta$.

28./XI. 4-10 cyl. Geb. fast in jedem Gesichtsfeld. Sonst St. idem.

3. Tour beendet.

30./XI. Nur homogene cyl. Geb. in gr. Menge. Schleimfäden, Schleimcylindroide. 
2.XII. Nach 20 Inunktionen symptomlos entl.

Nr. 8. C. 19jähr. Tischler, aufgen. am 14./XI. Trinkt bloB an Sonntagen große Mengen Biers. Lues cutanea macul. papul. Pap. seb. capill. Pap. exulc. tonsill. Pap. exulc. penis et scroti. Inf. Juni 1905. Scleraden. univ.

15./XI. Keine Cyl. Reichl. Leuk. Epithelien versch. Art.

16./XI. St. idem, E. $\varnothing .1025$.

18./XI. Ein fein granul. dünner Cyl. Massenhafte Ep. und Leuk. Heute 3. Inunktion. Oxalsauerer Kalk. Krist. sehr reichlich.

21./XI. Nach d. 5. Inunktion. In mehreren Gesichtsfeldern 1-3 hyal. cyl. Geb. Epith. und Leuk. in mäßiger Zahl. E. $\theta$. sp. G.?

23./XI. Ziemlich häufig in den Gesichtsfeldern. bis 8 homog. cyl. Geb. kleine, beiders. abgestumpfte Formen überwiegen. Massig Schleimfäden. Plattenep. überwiegen die Leuk.

26./XI. Fieber 39·2, Darmspülung.

27./XI. $37 \cdot 6,37 \cdot 2$.

28./XI. $36 \cdot 9$.

29./XI. Hg-Kur wieder aufg. Typ. Befund. sp. G. 1016. E. $\emptyset$.

30./XII. Starke Stomatitis.

2./XII. Entl. nach 2 Touren.

Nr. 9. J. 26j. 'Tischlergehilfe. Aufgen. 16./IV. Kein Alkoholiker (3 Glas Bier täglich.) L. cut. mac. papulosa. Pap. penis et scroti. Sklera. denitis univ. Cicatrices arcus palatogloss.

Inf. März 1905. Im Juni erstes Exanthem. Eine Tour geschmiert. Seit einer Woche das Rezidiv.

17./XI. Massenhafte Oxalsäurekristalle. Leuk. wenig Epith. 2zyl. Geb. 1030 E $\theta$.

18./XI. Ein hyal. Zyl. mit einem Lenkocyten, Epithel vorwiegend Übergangsep. (Nierenep.?) Leukocyten, Oxalatkristalle spärlicher als gestern.

20./XI. Nach d. 4. Inunktion: Ca, 1-4 homog. cyl. Geb. in vielen Gesichtsfeldern. Epithelien nicht sehr reichlich. sp. G. 1020. H $\theta$ Fe. Ess. $\theta$. Sp. schwach + , keine Oxalsäurekristalle.

24./XI. E $\theta$. 1027. Status idem.

28./XI. 2-10 cyl. homog. Gebilde in fast jedem Gesichtsfeld. Ein inkrust. hyal. br. Cyl. Agglomerationen v. roten, ausgelaugten Blutkörp. $\mathrm{E} \otimes 1025$. entlassen.

30./XI. Pat. wird nach d. 2. Tour auf eigenen Wunsch symptomfrei

Nr. 10. 36j. Kutscher, aufgen. 13./XI. Trank bis $40 \mathrm{Gl}$. Bier täglich. Phimosis inflam. Balanoposthitis, Skleradenitis inguin. Cu.-Spülung.

24./XI. Am Stamme deutliche Roseolaflecken.

25./XI. F. essig. $\theta$ H Sp. + ? Reichliches Sediment, Leukocyten weniger Epith., keine Cylinder.

26./XI. 1. Inunktion.

27./XI. Keine Cyl. Gebilde 1022, E (1-3) $\theta$ einzelne Spermatozoen. 
1./XII. Nach d. 5. Einreibung ca. 10 Cyl. Geb. in 3 u. 4 Präp. in den ersten zwei weniger E. $\theta$ amorphe Salze.

2./XII. Nach d. 6. Einreibung in 4 Präparaten 8-12 homogene cyl. Gebilde. Einzelne Schleimcylindroide, Epith, und Lenk. wie gewöhnlich, Schleimfäden. E $\theta$. sp. G. $102 c$.

Nr. 11. E. 2Rj. Schneidermeister, aufgen. am 27./XI. Kein Alkoholiker. L. cut. ulcerosa serpiginosa. Pap. muc. oris et tonsill. Pap. exulc. capill. Skleradenitis univ. Cicatrix ad frenulum post sclerosim.

Außer der Hg-Kur ca. $1 / 2 m$ gr. Pflaster auf die vle. Stellen.

L. extramat. Coitus im Dez. 1904. Im Februar 19052 Touren. Husten, Schlingbeschw. pap. Exantbem, im April nochmals 2 Touren. Ende September 1 Inj. Im ganzen ca. 10 Touren geschmiert.

28./Xl. Schleimfäden, Schleim-Cylindroide wenig Formelem. Leuk. und Epith. 2 hyal. cyl. Geb. E $\theta$. 1025. 1. Inunktion.

29./XI. 2 hyal. Cyl. im 2. Präparat; wenig Formel. Leuk. u. Epith. E $\theta$. sp. G. 1027.

1./XII. Nach d. 3. Inunktion. Vereinzelte cyl. Gebilde, ca. 4 in einem Präp. E $\theta$. sp. G.? Sonst mehrere Schleim-Cylindroide etc. Nephritis.

4./XII. Ca. 6 kleinere cyl. Gebilde, ein breiter hyal, wie bei

12./XII. Nach der 2. Tour. Eine Menge cyl. Geb. verschiedenster Form im Gesichtsfeld, sonst wie gewöhnlich. E $\theta 1020$.

14./XII. Geb. entlassen.

Nr. 12. Je. 49jähriger Schuhmacher, aufgen. 2./XII. Kein Alkoholiker. Lues cutan. papulosa, Sclerosis in sanatione, Skleradenitis praecipue inguin. Inf. angeblich vor 4 Wochen. Seit 10 Tagen besteht das Exanthem.

4./XII. Erste Inunktion.

6./XII. In jedem d. 4 Präp. 2 homog. cyl. Gebilde, eins inkrustiert. Zahlr. Schleimfäden. E $\theta .1028$.

7./XII. Nach d. 3. Inunktion neben reichl. Schleimfäden in zahlr. Gesichtsfeldern 1-2 Cyl. Geb. (typisch für Hg-Cylindrurie) 2 granul. Cyl. Ein. mit Leuk. bs. Cyl, reichliche Oxalsäurekristalle.

8./XII. Oxalsäure $\theta$. Cyl. Gebilde weniger als gestern.

9./XII. In vielen Gesichtsfeldern 1 cyl. Gebilde.

15./XII. Spärliche granul. Cylinder, reichl. cyl. Geb.

19. und 20./XII. Stomatitische Beschwerden.

22.-28./XII. 3. Tour. Stets der typ. Befund.

28.- entl.

Nr. 13. Ci., 27j. Diener, aufgen. 21./XI. 10-12 Gl. Bier täglich. L. cut. pap. Pap. seh. cap. Angina follic. Gingivitis. Stomatitis levis gradus. Psoriasis plant. Sclerad. praecipue inguin. Scleros. ad orif. ext. urethrae.

Vom 22.-25./XI. fieberhafte Angina. Epithelien.

22./XI. E +. (schwach) 2 hyal. Cyl. Schleimcylindroide. Leukocyten. 
25./XI. BloB Sp +. 1034. Erste Inunktion.

27./XI. Dickes gallertiges Sediment. Keine Cyl. Schleimcylindroide. Epith. Leukoc.

30./XI. In mehreren Gesichtsfeldern die typ. Gebilde. Plattenep. Epithelkonglomerate. Leukocyten.

1./XII. Stomatitis, entlassen.

Nr. 14. Wa., 33j. Witwer, Hühneraugenoperateur, aufgen. 26./XI. Früher Alkoholiker, bis 20 Gl. B. tgl. Lues cut. mac. papulosa. Papulae seborrh. faciei. Pap. muc. oris., labii inf. scroti et penis exulc. Alopecia luetica. Scleradenitis univ. praecipue inguin. Hyperkerat. plant. et. palmaris luetica.

27./XI. Schleimf. Plattenep. Leukoc. Keine cyl. Gebilde. E $\theta .1025$.

29./XI. Status idem, spärliche Schleimzylindroide.

2./XII. E $\theta$. 1025. Fin langer hyal. mit Leuk. bes. Cylinder, sonst St. idem.

6./XII. Ein br. hyal. Cyl., 2 hyal. Kugeln. Plattenep. Nierenep.?

9./XII. Nach der 10. Inunktion: reichl, cyl. homog. Gebilde, E $\theta$.

1015. Sonst Status idem. Dichtes Gewirr von Schleimfäden.

Nr. 15 siehe im Text.

Nr. 16. Bo., 51j. Taglöhner, aufgen. 14./XII. Stupides Indiv., anamn. nichts zu eruieren. Lues gummosa. Dermatitis ex combustione gr. II. Eine größere Menge gr. Pflasters zur Deckung der ulz. Gummen.

18./XII. 1. Inunktion.

21./XII. E $\theta$. sp. G.? Leukoc. gesch. Plattenep. Übergangsep. sehr reichlich. Detritus. Schleimfäden. Keine Cyl.

27./XII. Nach d. 7. Einreibg. E Ө.sp. G. 1015. Cyl. Gebilde in mehreren Ges.-Feldern in den verseh. Formen. Stomatitis.

28./XII. Nach d. 8. Einreibg. 2-4 zyl. Geb. in vielen Gesichtsfeldern, wenige Schleimcylindroide. Ep. u. Leuk. reichlich, doch spärlicher als gestern.

30./XII. Fe. Essigs. +. Sp +. H O. Sehr reichl. cyl. Gebilde in mannigfaltigsten Formen (ausgesetzt $\mathrm{mit} \mathrm{Hg}$ ). feldern.

23./I. 06. Wieder untersucht. Zyl. noch reichl. 1-2 in viel. Gesichts-

Nr. 17. H., 21j. Beamter, aufgen. 22./XI. Kein Alkoholiker. (1-2 Gl. Bier täglich.) Lues cut. pap. Pap. mucosae oris, labiorum, tonsill. hypertr. Papul. in regressu faciei. Erosiones ad anum. Scleraden. praecipue retro et submax. Sclerosis sanata orif. ext. urethrae.

Inf. vor 4 Monaten.

Konstit. Symptome vor $1 \frac{1}{2}$ Mon. 3 Touren gesehmiert.

22./XI. 1. Inunktion.

23./XI. 2. Inunktion, sp. G. 1025. Fe. Essigs, schwach +. Sp. schw. po. H schw. †. Reichl. Schleimcylindroide, Schleimfäden, Epithelien, wenig. Leukoc.

24./XI. Viel Schleimfäden. Ein Cylinder? Fe. Essigs. $\theta$. Н $\theta$ Sp. $\theta$. sp. G. 1016.

Arch. f. Dermat. u. Syph. Bd. LXXXIII. 
29./XI. E $\varnothing$. 1018. Wenig Sediment. (Klin. Zentrifuge.)

$$
2 \text { hyal. Cyl. }\left\{\begin{array}{l}
1 . \text { Präp. } \\
2 .
\end{array}\right.
$$

4./XII. Cyl. Geb. bis 12 im Gesichtsfeld, mindestens 4 in jedem Gesichtsfeld. E $\theta .1020$.

5./XII. Symptomlos nach 2 Touren entlassen.

Nr. 18. Š., 24j. Tischler, aufgen. 28./XII. Kein Alkoholiker. Lues cut. mac. papulosa. Pap. seb. capillitii. Corona venerea. Pap. muc. oris, labiorum, tonsill. Keratosis plant. et palm. Pap. exulc. scroti et penis et ad anum. Sclerad. univ. Sclerosis sanata. behandelt.

Inf. August 1903. Erscheinungen seit September. Bisher nicht

29./XII. Keine cyl. Gebilde.

3./I. Nach d. 6. Inunktion: cyl. Gelsilde ziemlich reichlich. (In mehreren Gesichtsfeldern 1-2 cyl. Geb.)

4./I. Harn in 2 Portionen aufgefangen. In der 2. Portion mehr cyl. Geb. als in der ersten. In vielen Gesichtsfeldern 1-2 cyl. Geb. E $\theta$. Ep. u. Leuk, in mäßiger Zahl. Schleim. Detritus.

5./. Status idem, auch bez. des Cylindergeh. d. beiden Portionen. 11./I. Nach 14. Einreibg. reichl. Cyl.

Nr. 19. P., 25j. lediger Schneider., aufgen. 12./XII. Trinkt höchstens

2-3 Gl. Bier täglich. Inf. Jänner 1904.

März 19044 Touren, 2 Injektionen.

Nach $1 / 2$ J. 5 Touren.

Nach 5 Monaten 5 Welandarsche Säckchen.

L. cut. maculosa. Pap. seb. capill. Epitheliosis linguae, arc. palatogl. tonsill. sin. Epididymitis sinist. Scleraden. univ. Pap. scroti.

14./XII. Epithelzellen mehr als Leuk., beide nicht reichlich. Wenig Schleim. E $\theta$. sp. G.?

15./XII. Sehr reichl. Leuk., mehr als Epithelien. Wenig Schleim u. Detritus. Ein breiter hyal. Cylinder. E $\theta .1031$.

18./XII. Hente 6. Inunktion. E $\theta$. 1022. Klare Sedimentflüssigkeit mit wenig Flocken, Epithelien reichlicher als Leuk., spärlich Schleim.

19./XII. Trotz gen. Unters. ungef. und gefärbten Sediments sind cyl. Gebilde nicht zu entdecken. Sonst St. idem. Amorphes harns. Na. E $\varnothing .1028$.

27./XII. In ziemlich zahlreichen Gesichtsfeldern 1-2 homog. cyl.

Geb. Ep. Leuk. Schleim. Detritus. (Verwechslung des Harns!!? Siehe Anm. zur Tabelle S. 383.)

28./XII. Kleine Flöckchen im klaren Harn als Sedinent. Epith. Leuk. in mäßiger Menge. Ke in e cyl. Gebilde.

29. u. 30./XII. Leuk. Epithelien. Wenig Schleimfäden. Keine Cylinder und keine Cylindroide. 1017. E $\theta$.

Nr. 20. K., 28j. Ökonom., aufgen. 3./XII. Kein Alkoholiker.

Infektion unbekannt. (Vieileicht vor 2 Jahren durch einen syph. (?) Knecht.) Frau und Kinder gesund. 
Im März 19054 Touren geschmiert.

Luev gammosa, Gummöse Periostitis d. 1. Stirnhöckers. Fast vollständiges Fehlen des knorpeligen und knöchernen Nasenseptums. Gumma d. 1. Gaumenbogens im Zerfall. Verwachsung d. r. Gaumenbogens mit d. hint. Pharynxwand. Ausgebr. Narben u. serpiginöse Infiltrate am Stamm u. d. Extremitäten.

Inunktionen à $4 g$ Airol., gr. Pflaster. Zittmans J.-K,, weiße Praecipitatpomade, Jodtinktur.

4./XII. Reichl. Schleimfäden, lange, bandartige Schleimcylindroide. Wenig zellige Elemente. Leuk. u. Epith. 1030. E $\theta$.

5./XII. Sp. +. 1028. Status idem.

7./XII. In jedem Präparat ein hyal. Cyl. Dichtes Netz von Schleimfäden, Sp. + . Kochprobe $\theta$. Fe. Essigs. $\theta$. H $\theta$.

11./XII, Spärlich Epith, u. Leuk. E $\varnothing$. 1015. Reichliche cyl. Gebilde in mannigfaltigen Formen.

12. u. 13./XII. Typ. Befund.

14./XII. Typ. Befund. Sp. +. 1020.

15./XII. Sehr reichl. homog. cyl. Geb. 1022 . E (1-3) $\varnothing$.

16 /XII. Homog. cyl. Geb. 1018. Sp. †. E $(1,2) \theta$.

21./XII. Stomatitis, die bis zum 4./XII. allmählich abklingt.

25.-31./XII. Erysipel, faciei. Hg-Kur ausgesetzt.

31./XII. Typ. Befund. Doch geringere Zahl von cyl. Geb. als früher. 6.-13./I. 3. Tour. Gr. Menge v. cyl. Gebilden.

13./I. Gebessert entlassen.

Weiber:

D., 18j. Kellnerin, aufgen. 12./XII. Trinkt über einen Liter Wein täglich. L. c. Pap. luxur. tons. Pap. exulc. ad genit. ext. et nymphas. Scleraden. praecipue inguin. Ulcera multiplicia.

15./XII. Vaginalep., Leukoc. ziemlich reichlich. Schleimfäden. Schleimcylindroide. E $\varnothing$. sp. G.?

19./XII. E $\varnothing$. sp. G. 1016. Spärliche Krist. v. oxalsaurem Kalk. Sonst St. idem.

Vom 18./XII._26./XII. Stomatitis.

28./XII. 4 hyal. cyl. Geb. im Präparat. 8. Einreibung.

31./XII. 2. Tour beendet.

2./I. Über 12 cyl. Gebilde im Präp.

10./I. 19. Einreibg. 2-4 cyl., vorwiegend kleine Geb. in vielen Gesichtsfeldern.

11./I. Vormittagsharn 8-1 Uhr gehalten: spärliche cyl. Gebilde.

M., 18j. Schneiderin, aufgen. 12./XII. L. cut. mac. Pap. ad tons. hypertr. ad genit, extern. maceratae partim exulc., confluentes. Ekzema intertrigo. Sclerad. univ.

Erster Spitalsaufenthalt: Ulcera multiplicia $15 . / \mathrm{XX}-16 . / \mathrm{X}$.

13./XII.-16./XII. Fieberhafte Angina.

16./XII. u, 27./XII. Je ein cyl. Gebilde. 
21./XII. 2-3 cyl. Gebilde, Schleimfäden, lange, bandartige Schleimcylindroide. Epith. u. Leukoc.

28./XII. Erst nach Verdünnung mit phys. NaCl-Lösung. In mehreren Gesichtsfeldern bis 4 cyì. Gebilde (versch. Formen).

11./I. Nach der 3. Tour.

In vielen Gesichtsfeldern 4 (in einigen bis 6) cyl. Gebilde

Im Vormittagsharn nur spärliche cyl. Gebilde.

Auf die Wiedergabe der Protokolle von den bloß zu Kontrollstichproben verwendeten Patienten muf ich der gebotenen Raumbeschränkung wegen verzichten und aus demselben Grunde war die ausführliche Mitteilung aller Einzeluntersuchungen bei den protokollierten Fällen nicht möglich.

\section{Literatur.}

Brugseh, Th. Salizyltherapie und Nieren. Die Therapie der Gegenwart. 1904. p. 58.

Bürgi. Größe und Verlanf der Quecksilberausscheidung durch die Nieren bei den verschiedenen üblichen Kuren. Arch. f. Derm. u Syph. Bd. LXXIX. Heft 1 und 2.

Elbe. Die Nieren- und Darmveränderungen bei Sublimatrergiftung des Kaninchens und ihre Abhängigkeit vom Gefäßsystem. Virchows Archiv. Bd. CLXXXII. p. 485.

Fürbringer. Über Albuminurie durch Quecksilber und Syphilis. Verh. d. Kongr. f. innere Medizin. 4. Kongr. Wiesbaden. 1885.

Gentzen. Über Cylindrurie und Nephritis. Deutsche medizinische Wochenschr. 1905. Nr. 33.

Glaser. Über den Einfluß alkoholischer Getränke auf das Harnsediment des normalen Menschen. Deutsche med. Wochenschrift. 1891. Bd. XVII. p. 1193.

Gabritschewsky, G. Über die Untersuchung des Sputums in Schnitten etc. Deutsche med. Wochenschr. Bd. XVII. p. 1198. 
Gravagna. Sulle altera zioni anatomiche renali in seguito a dosi terapentico di Mercurio. Giorn. ital. delle malattie venere etc. Bd. XXXIV. p. 503. Ref. Archiv f. Derm. u. Syph. Bd. LX. p. 312.

Heller. Über merkurielle Albuminurie. Charité Annal. Bd. XX. 1895. p. 701-717 und Berliner klin. Wochenschr. Jg. XXXII. (1895.) p. 994. Berl. med. Gesellschaft. Sitzung vom 23. Oktober 1895.

Karvonen, J. Über den Einfluß des Quecksilbers auf die Nieren. Dermatol. Zeitschr. Bd. V. 1898. p. 113.

Klemperer. Ủber die Veränderung der Nieren bei Sublimatvergiftung. Virchows Archiv. CXVIII. 445.

Klieneberger und Oxenius. Über Urine und Urinsedimente bei normalen Personen, bei rheum. Erkrank. und nach der Einwirkung von Salizylpräp. Deutsches Archiv für klin. Medizin. Bd. LXXX. 1904. pag. 225.

Klieneberger. Über Urine und Urinsedimente bei chr. und lok. Staungen, in Endzuständen und im Kollaps. Münch. med. Wochenschrift. 1905. Nr. 25, 26, 27.

Kobler. Beiträge zur Kenntnis der Nierenerscheinungen bei akuten Darmaff. nebst Bemerk. über die Bildung der hyalinen Cylinder. Wien. klin. Wochensehr. 1870. p. 531.

Lewin. Berliner klin. Wochenschr. Jg. XXXII. 1895. pag. 971. Diskussion über den Hellerschen Vortrag. Daselbst auch Diskussionsbemerkung von Fürbringer.

Litten. Die Zentrifuge im Dienste der klin. Medizin. Deutsche med. Wochenschr. 1891. Jg. XVII. p. 749.

Lüthje. Über die Wirkung von Salizylpräparaten auf die Harnwege nebst einigen Bemerkungen ủber die Genese der Cylinder und Cylindroide. Deutsches Archiv f. klin. Mediz. Bd. LXXIV. p. 163.

Overbeck, R. Merkur und Syphilis. Berlin. 1861.

Ros en heim. Zur Kenntnis der diuret. Wirkung der Hg-Präparate. Deutsche med. Wochenschr. 1887. p. 325. (R. sah bei innerl. Anwendung des Kalomel bei Hydropsien niemals Eiweiß oder Formelem. im Harn auftreten.)

Schwimmer. Über das Vorkommen der Albuminurie bei luetischen Affektionen. Wiener mediz. Wochenschr. 1892. p. 1913.

Török und Pollak. Über die Entstehung der homogenen Harncylinder und Cylindroide. Archiv für experim. Pathologie und Pharmakol. Bd. XXV. p. 87.

Welander. Über Albuminurie und Cylindrurie durch Syphilis und Quecksilber. Nord. Med. Arkiv. Bd. XXXIII. Nr. 29.

- Kann die Beh. mit Quecks. Cylindrurie und Albuminurie hervorrufen. Arch. f. Derm. u. Syph. Bd. XXVI. p. 331.

- Klinische Studien über Nierenaffektionen bei Syphilis. Arch. f. Derm. u. Syph. Bd. XXXVII. p. 91, 323 .

- Einige Worte über die Form der Anwendung des Quecksilbers. Arch. f. Derm. a. Syph. Bd. XLVI. p. 39. 
Winternitz. Quantitative Versuche zur Lehre über die Aufnahme und Ausscheidung des $\mathrm{Hg}$. Archiv für experim. Path. etc. Bd. XXV. p. 225.

- Über die Ausscheidungsgröße des Hg. Archiv für Dermatologie und Syphilis. Bd. XXI. 1889. p. 783.

Unbekannter Verfasser: Albuminurie und Cylindrurie infolge von Syph. und Hg-Behandlung. Journal de practic. 1900. Nr. 52. Ref. nach Un na XXXII. p. 640 . 